\title{
ARTICLE
}

\section{The neuropsychiatry of multiple sclerosis}

\author{
Maria A. Ron
}

\begin{abstract}
Maria A. Ron is emeritus professor of neuropsychiatry at University College London, Institute of Neurology. Professor Ron's research interests are the cognitive and psychiatric manifestations of neurological disease and structural brain abnormalities in psychoses.

Correspondence Professor Maria A. Ron, 24 Claylands Road, London SW8 1NZ, UK. Email: m.ron@ucl.ac. uk
\end{abstract}

\section{Copyright and usage}

(C) The Royal College of Psychiatrists 2018

\section{SUMMARY}

Multiple sclerosis (MS), an immune-mediated demyelinating condition, is the most common neurological disease affecting young adults in the UK. It has a high psychiatric comorbidity and over half of patients have some degree of cognitive impairment that adds to the burden of disability. This article reviews the psychiatric and cognitive manifestations of MS and their detection and treatment. Recent advances in the treatment of the disease are briefly reviewed and the impact of disease-modifying therapies on psychiatric morbidity and cognitive impairment is discussed.

\section{LEARNING OBJECTIVES}

- Understand the psychiatric morbidity in MS and its biological counterparts

- Understand the cognitive impairment and its biological counterparts

- Become familiar with the detection and treatment of the psychiatric and cognitive manifestations of MS

\section{DECLARATION OF INTEREST}

None.

Multiple sclerosis (MS) is an immune-mediated, demyelinating neurological disease with a chronic course (Box 1). In the UK, MS is the most common neurological disease in young adults. It affects an estimated 2.5 million people worldwide. MS is more common in women and in White populations living in northern latitudes. Psychiatric comorbidity is common in MS. Cross-sectional studies of hospital populations have reported symptoms of depression and anxiety in nearly half of patients. In population-based surveys the prevalence is closer to $25 \%$ (Marrie 2015). Depression and anxiety decrease quality of life and adherence to disease-modifying therapies (DMTs).

Cognitive impairment can be detected in about $40 \%$ of people with MS and can be present in the early stages of the disease, although it becomes more severe as the disease progresses and enters its chronic phase. Memory, speed of information processing and executive function are most impaired, while simple attention and language tend to be better preserved.

\section{Multiple sclerosis: the disease}

The mean age at MS onset is around 30, and the female:male ratio is $3: 1$. In $85-90 \%$ of cases MS starts with an episode of acute neurological symptoms known as a clinically isolated syndrome (CIS). Optic neuritis, myelitis or brain stem involvement are the most common CISs. Symptoms peak within 4 weeks and there is complete or partial remission. Most patients follow a relapsing-remitting course within 5 years of the CIS (relapsingremitting MS or RRMS). Some patients follow a benign course with little disability. Permanent disability occurs when there is incomplete recovery from relapses or when the disease enters its progressive phase (secondary progressive MS or SPMS), usually 15 to 20 years after disease onset. In a small proportion of patients (15\%) the disease follows a progressive course from the beginning (primary progressive MS or PPMS). The mean age at onset for PPMS is 40, and the most common presentation is an asymmetrical paraparesis. MS reduces life expectancy by a median of 7 years.

The cause of MS is uncertain. The increased incidence of MS in first-degree relatives and the concordance in monozygotic twins (around 30\%) suggest a genetic component. More than 50 susceptibility loci, many closely mapped to immunologically relevant genes, have been identified in genome-wide association studies. Possible environmental factors interacting with the genetic predisposition include Epstein-Barr virus infection, vitamin D deficiency, latitude and smoking.

\section{Neuropathology of MS}

Acute MS relapses are characterised by active, inflammatory, focal demyelinating white matter lesions commonly in the optic nerve, periventricular white matter, corpus callosum, brain stem, cerebellum and cervical cord. As the disease enters a progressive course, the acute inflammatory reaction is less marked and widespread neurodegenerative changes (diffuse axonal damage, microglial activation and cortical demyelination) become more prominent. Damage to white matter tracts results in 
BOX 1 Multiple sclerosis (MS)

- MS is an immune-mediated demyelinating disease

- It is more common in women

- The mean age at onset is around 30

- In $75 \%$ of patients, MS has a relapsing-remitting course and enters a progressive phase 15-20 years from onset

- Life expectancy is reduced by about 7 years

- Acute relapses are characterised by inflammation and demyelination, and lesions occur in white and grey matter of the brain and in the spinal cord

- Neurodegeneration occurs in parallel

cortical atrophy, prominent in the cingulate and temporal cortices and in other interconnected cortical regions. It is unclear whether acute inflammation triggers the neurodegenerative changes or whether the two occur independently of each other.

Magnetic resonance imaging (MRI) has played a crucial role in advancing the understanding and treatment of the disease by making it possible to study MS pathology in vivo. MRI visualises macroscopic lesions in white and grey matter (Fig. 1). Of these, T2 hyperintensities represent heterogeneous pathology (oedema and inflammation, demyelination and remyelination, gliosis and axonal loss), whereas T1 hypointensities represent demyelination and axonal loss. Using diffusion-based techniques such as diffusion tensor imaging (DTI), and other techniques such as magnetisation transfer and spectroscopy, subtle abnormalities in the 'normalappearing brain tissue' (NABT) can be detected and quantified. Abnormalities in the individual tracts connecting different brain regions can be studied using tractography, another DTI-based technique. It is also possible to quantify white and grey matter atrophy.

\section{Diagnosis}

Early diagnosis is increasingly important as DMTs are more effective when used early in the disease. The diagnosis of MS requires evidence of lesion dissemination in space and time, and MRI is used to provide this evidence. Dissemination in space requires lesions in at least two of four areas (optic nerve, periventricular, juxtacortical or spinal cord) and dissemination in time requires new lesions in two consecutive scans or enhancing (acute) and non-enhancing (chronic) lesions in the same contrast (gadolinium) enhanced scan. In established MS, the brain MRI is always abnormal and spinal cord lesions are also present in 90\% of cases. Cerebrospinal fluid (CSF) examination is no longer necessary to make a diagnosis if the clinical picture is in keeping with the MRI findings. The CSF usually shows mildly raised white cell count (by up to 25 cells $/ \mathrm{cm}^{3}$ ), raised protein (by up to $1 \mathrm{~g} / \mathrm{L}$ ) and immunoglobulin G (IgG) index. In $90 \%$ of cases IgG oligoclonal bands are present in the CSF, but not in serum.

Solomon and colleagues have reported that MS may be misdiagnosed in as many as $10 \%$ of patients, a significant proportion of whom have functional neurological disorders. A quarter of these patients were unnecessarily exposed to DMTs (Solomon 2013).

\section{Treatment}

All available DMTs for MS act via immunomodulatory and immunosuppressive mechanisms and they improve the course of RRMS by reducing the relapse rate, and the lesion burden and brain atrophy detected by MRI. The first DMT, interferon

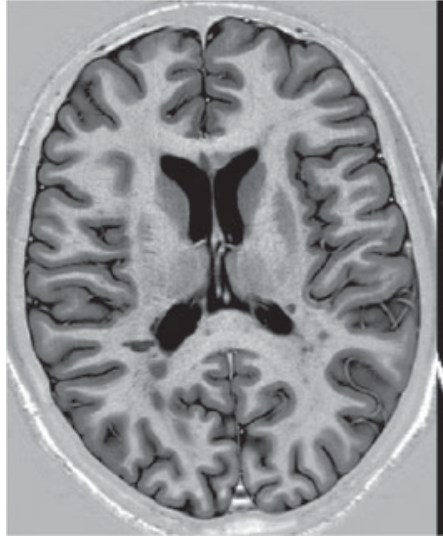

PSIR

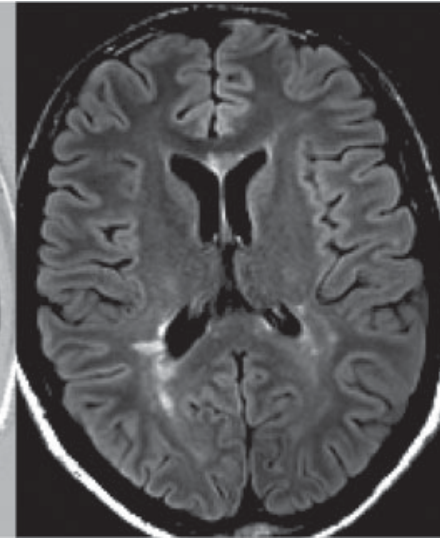

FLAIR

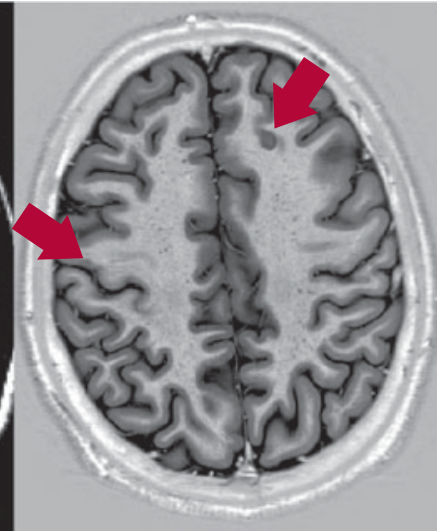

PSIR

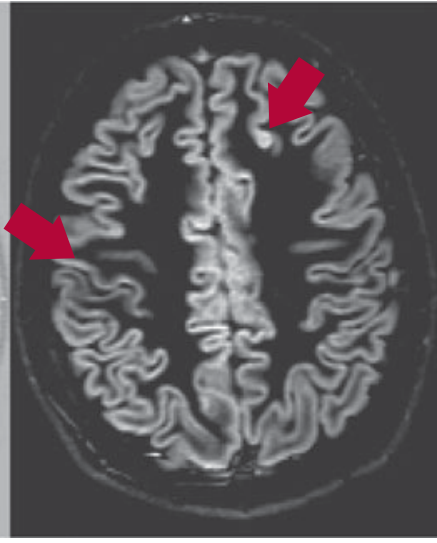

DIR

Cortical and periventricular lesions in multiple sclerosis, identified by three magnetic resonance imaging techniques: PSIR, phase-sensitive inversion recovery; FLAIR, fluid-attenuated inversion recovery; DIR, double inversion recovery. 
beta, became available in the 1990s and other interferon-beta related drugs and glatiramer acetate soon followed. Early DMTs were injectable drugs of limited efficacy and low adherence. More effective second generation DMTs include fingolimoid and several humanised monoclonal antibodies (e.g. natalizumab, alemtuzumab, ocrelizumab) that are administered intravenously. These more effective compounds also have severe side-effects, including autoimmunity and progressive multifocal leukoencephalopathy (PML), a CNS infection caused by the John Cunningham virus (JCV). These DMTs, except for ocrelizumab, are not effective in progressive MS, where neurodegeneration has an overriding role. It is uncertain whether DMTs modify the risk of conversion to SPMS or the length of the transition period.

DMTs should be used early in the disease to control the initial inflammatory component. The escalation method of treatment advocates the initial administration of low-risk DMTs (e.g. interferon-beta or glatiramer acetate), followed by higher-efficacy DMTs (e.g. natalizumab, alemtuzumab or fingolimod) if the former prove to be ineffective or intolerable. Patients with severe MRI abnormalities at presentation (including brain atrophy) and a clinically aggressive course may benefit from the induction treatment method that uses high-efficacy DMTs for 1 or 2 years, followed by an observation period or by a low-risk drug.

The use of neuroprotective agents (e.g. glutamate antagonists, sodium channel blockers and cannabinoids) in MS is a promising field, but the possible effects on cognition remain to be determined.

\section{Prevalence and incidence of psychiatric morbidity}

Psychiatric abnormalities in patients with MS were documented by Charcot in 1877. In the UK, Cottrell and colleagues, in an early impressionistic study, reported depression, euphoria (a prevailing mood of serenity and cheerfulness), eutonia (a sense of physical well-being) and abnormal emotional expression (lability of affect) in patients with well-established MS (Cottrell 1926). The prevalence of these abnormalities has recently been well documented by Marrie and colleagues (Table 1), in a systematic review of 118 population-based studies (Marrie 2015). However, few of these studies included control populations or documented the incidence of psychiatric morbidity.

\section{Depression and anxiety}

In population-based studies, anxiety and depression have been detected in over $20 \%$ of people with MS, often at the time of the initial diagnosis, although
TABLE 1 Reported prevalence of psychiatric morbidity in multiple sclerosis (MS)

\begin{tabular}{|lc|}
\hline Disorder & Prevalence, \% \\
\hline Depression & 23.7 \\
Anxiety & 21.9 \\
Alcohol misuse & 14.8 \\
Bipolar disorder & 5.8 \\
Psychosis & 4.3 \\
Substance misuse & 2.5 \\
\hline
\end{tabular}

Source: adapted from Marrie et al (2015).

the prevalence rises as the disease progresses (Marrie 2015). Hospital-based populations have a reported prevalence of nearly 50\% for these disorders in community-dwelling patients with MS (Bamer 2008), higher than those in similarly disabled patients without neurological illness. Depression was associated with severe disability, poor educational background and lack of social support. Depression was mild in about half of the patients. Depression at illness onset often delayed the MS diagnosis, as physical symptoms were misattributed to depression.

A systematic review of causes of death in MS patients (Scalfari 2013) concludes that the frequency of suicide is significantly increased compared with the general population, but that its prevalence is more difficult to determine because of cultural differences and reporting bias. Feinstein and colleagues reported suicidal ideation in almost a third of a community sample of people with MS, and just over a fifth of those individuals had attempted suicide. Living alone, depression and alcohol misuse were predisposing factors (Feinstein 2002).

The course of depression in people with MS has seldom been assessed, but clinical experience suggests that it is variable. Ensari and colleagues, using the Hospital Anxiety and Depression Scale (HADS), followed up a large group of patients with RRMS over a period of 30 months. The overall group scores remained unchanged, but in a third of those with high initial scores, the severity of depression increased over time, whereas the remaining two-thirds it improved over the follow-up period. A good outcome was correlated with older age, being married and in employment, and with physical activity. Gender, race, degree of disability and duration of illness were not significantly related to the outcome of depression (Ensari 2014).

The relation between fatigue and depression in people with MS is a complex one. Fatigue may be present in euthymic patients, but depressed patients tend to be more fatigued and treatment of depression also results in some improvement of fatigue. 


\section{Aetiology of depression in MS}

Feinstein and colleagues have reviewed this topic in detail (Feinstein 2014). The relevance of genetic factors is still uncertain, as there is no conclusive evidence that depression is increased in relatives and, in contrast with non-neurological populations, its prevalence appears to be similar in men and women. Psychosocial factors (stress, social isolation and maladaptive coping mechanisms), on the other hand, are clearly relevant and may account for $40 \%$ of the variance.

Immunological factors and inflammation are increasingly recognised in the aetiology of depression in non-neurological populations. Pro-inflammatory cytokines that reduce synaptic serotonin release are known to occur in MS and to correlate with the severity of depression. The role of inflammation in depression in people with MS is still unclear, but depression may be correlated with the presence of acute lesions during relapses, and DMTs with anti-inflammatory effects may have a beneficial effect on depression.

Hyperactivity of the hypothalamic-pituitaryadrenal axis (HPA) is also present in MS, with failure to suppress cortisol in response to dexamethasone, particularly during acute relapses.

\section{Depression and brain pathology}

The correlation between MRI structural brain abnormalities and depression is not a close one and brain pathology only explains around $40 \%$ of the variance. Limbic pathology appears to be particularly relevant. Feinstein and colleagues reported a greater T2 lesion load in the left medial frontal regions, together with left temporal atrophy in depressed MS patients, and MRI diffusion abnormalities in the white and grey matter were also present in the left temporal lobe (Feinstein 2010). Nygaard and colleagues found reduced cortical thickness and volume in a group of 61 RRMS patients when compared with matched controls (Nygaard 2015). Symptoms of depression were also correlated with cortical atrophy in the frontal pole, orbitofrontal, mid-temporal, fusiform and parahippocampal regions in the same patients, and with anterior cingulate atrophy (Gobbi 2014). The relevance of these findings is strengthened by the correlation between structural brain abnormalities and high cortisol levels in depressed MS patients (Gold 2010).

Damage to white matter tracts connecting limbic structures results in altered connectivity between key components of these emotional networks, leading to abnormal processing of emotional stimuli. This has been illustrated by functional MRI (fMRI) studies that have reported abnormal connectivity between the amygdala, hippocampus and prefrontal regions in non-depressed MS patients (Passamonti 2009), and alterations in functional connectivity within the default mode network (Rocca 2015), that in turn correlate with the volume of white matter lesions. In parallel, tractography studies (Nigro 2015) have reported structural changes in the corresponding white matter tracts connecting the hippocampus, amygdala, ventrolateral and dorsolateral prefrontal cortices and supplementary motor areas in depressed MS patients.

\section{The diagnosis of depression}

The challenge of diagnosing depression in neurological patients is that of separating symptoms clearly attributable to depression from those due to the physical illness. MS symptoms also common in depression include fatigue, poor concentration and memory and sleep difficulties. Strober and colleagues (Strober 2010) used various questionnaires and informant interviews to identify a hierarchy of symptoms most useful in diagnosing depression in people with MS: this is outlined in Box 2.

Depression should be screened for in primary care or in neurology clinics, and the American Academy of Neurology has recommended the self-administered Beck Depression Inventory (BDI) to detect depression and the General Health Questionnaire (GHQ) for detecting more broadly defined emotional disturbances. In the UK, the HADS is commonly

BOX 2 Diagnosing depression in multiple
sclerosis

Most useful diagnostic symptoms

- Sadness

- Pessimism

- Sense of failure

- Feelings of guilt and disappointment

- Changes in appetite and weight

Useful only if severe

- Loss of interest

- Crying

- Dissatisfaction

- Irritability

- Self-criticism

Of little diagnostic value

- Loss of libido

- Fatigue

- Poor sleep

- Indecisiveness

(Strober 2010) 
used because it focuses on non-physical symptoms of depression.

\section{Treatment of depression and anxiety}

Depression and anxiety in MS are underdiagnosed and undertreated, despite their impact on quality of life. Lack of psychiatric resources and patients' reluctance to use them leave detection and treatment of depression in MS patients mostly in the hands of neurologists. Approximately half of MS patients with major depression attending neurological clinics may be left untreated and, when antidepressants are prescribed, doses are often suboptimal and there is frequently no monitoring of progress. (Mohr 2006).

Clinical experience suggests that depressed patients with MS may respond to conventional psychological and pharmacological treatments, but there is a paucity of well-conducted clinical trials to back this view. A systematic review and metaanalysis of 20 randomised controlled trials (RCTs) of antidepressants in neurological patients, that included two RCTs in patients with MS, reported remission twice as often when antidepressants (selective serotonin reuptake inhibitors (SSRIs) or tricyclics) were compared with placebo (Price 2011). Improvement was present at $6-8$ weeks and was still evident in patients followed up for more than 18 weeks. Data were insufficient to examine the effects of medication on cognition and social functioning. A more recent Canadian meta-analysis (Fiest 2016) of studies in patients with MS also reported improvement in depression in 10 RCTs using SSRIs or tricyclic antidepressants, although there was no comparable effect for anxiety or fatigue. In clinical practice, SSRIs are the antidepressants of choice because of their milder sideeffects, although tricyclics may be useful to relieve pain and bladder dysfunction. Serotonin-noradrenaline reuptake inhibitors (SNRIs) are useful if patients do not respond to SSRIs. Electroconvulsive therapy (ECT) is not contraindicated in MS and is an option for patients with treatment-resistant depression.

The effectiveness of psychological treatment has not been thoroughly investigated in people with MS, although the limited evidence suggests that it may be helpful, and cognitive-behavioural therapy (CBT) is the treatment recommended in the guidelines of the American Academy of Neurology (Minden 2014). In their meta-analysis Fiest and colleagues reviewed 10 RCTs of CBT and reported improvement in depression, but not in anxiety or fatigue (Fiest 2016), and another systematic review of 22 published treatment trials of CBT in patients with neurological disease including MS (Fernie
2015) also concluded that individual or group CBT improved depressive symptoms. The effect size was greater when CBT was compared with passive conditions (e.g. waiting list controls or treatment as usual) than with other treatments (e.g. relaxation, social discussion group), and it was unclear from these studies whether the improvement was maintained over time. The role of physical exercise in the treatment of depression in MS remains to be determined.

Early reports suggested that treatment with interferon-beta was linked to depression and suicide. More recent studies describing the results of large controlled trials have shown this not to be the case, and a history of depression, rather than receiving DMTs, is the best predictor of post-treatment depression. Similarly, there is no evidence to suggest that glatiramer acetate or the second-line DMTs cause depression or increase psychiatric morbidity.

\section{Psychosis}

The prevalence of psychosis is higher in people with MS than in the general population (Marrie 2015). A Canadian epidemiological study (Patten 2005) reported a prevalence of 2-3\%. Differences in prevalence compared with the general population were more marked $(4 \%$ v. $0.5 \%)$ in those aged between 15 and 25 and the differences were no longer significant in those aged 65 or over.

Feinstein and colleagues compared 10 MS patients with psychosis with an age-, gender- and disability-matched group without psychosis (Feinstein 1992). The MRI lesion burden was greater in the psychosis group, particularly around the temporal horns. The mean age at onset of psychosis was 39.6 years, later than in 'primary' schizophrenia, and in nine out of ten patients neurological symptoms preceded psychosis by an average of 8.5 years. The onset of psychosis coincided with an MS relapse in only four patients. Symptoms were similar to those of schizophrenia. Persecutory delusions were most common (70\%), followed by passivity phenomena (30\%), thought disorder (20\%) and third-person hallucinations (20\%). Symptoms improved in response to antipsychotic medication. One patient followed a chronic course from the beginning, but for the rest the psychosis remitted after several weeks.

\section{Aetiology of psychosis in MS}

Several aetiological factors may be relevant. There are shared risk loci, mainly in the major histocompatibility complex (MHC) region of chromosome 6 (Andreassen 2015), between MS and schizophrenia, 
but not bipolar disorder, suggesting that abnormal myelination may be relevant in both.

Autoimmune mechanisms may also be relevant: compared with the general population, schizophrenia is associated with a $50 \%$ increase in life-time prevalence of autoimmune disease, and there is an increased familial association between autoimmune disease and schizophrenia (Benros 2014). Titulaer and colleagues described 23 out of 691 patients with autoimmune encephalitis (anti- $N$-methyl-Daspartate receptor or anti-NMDAR encephalitis) who had clinical and MRI features of acute demyelinating encephalomyelitis (ADEM), neuromyelitis optica or MS and it has been suggested that psychosis in people with MS and other demyelinating diseases, particularly in the presence of extrapyramidal features, may be caused by anti-NMDAR encephalitis. The latter diagnosis may be missed, and the relevant immunosuppressant treatment delayed, if psychotic and extrapyramidal symptoms are attributed to a demyelinating disease (Titulaer 2014).

\section{Treatment of psychosis}

There are no RCTs dealing with treatment of psychosis in people with MS. Clinical experience suggests that a good response can be achieved using atypical antipsychotic agents, although care is needed to minimise extrapyramidal and other unwanted sideeffects.

\section{Abnormalities of affective expression}

Abnormalities of affective expression are present in $10-30 \%$ of people with MS. These abnormalities, known as pathological laughter and crying, emotional lability or pseudobulbar affect (PBA), are characterised by repeated episodes of laughter or crying in response to non-specific stimuli that are incongruous with the underlying affect. PBA tends to appear in patients with advanced disease and disability. PBA is associated with deficits in processing speed, visual memory and executive function and with lesion burden in the brain stem, left parietal and bilateral medial frontal regions, suggesting that damage to widely dispersed neural networks is present.

The Center for Neurologic Study Lability Scale (CNS-LS) is a useful diagnostic tool. Clinical experience suggest that antidepressants and anti-epileptic medications are useful in the treatment of PBA, although little supporting data are available. More recently, dextromethorphan/quinidine (DM/Q) has proved to be effective and well tolerated (Pioro 2014). Dextromethorphan is an NMDA receptor antagonist with serotonin and noradrenaline reuptake inhibition properties, and the addition of quinidine increases its bioavailability.

\section{Cognitive impairment}

Cognitive impairment adds to the burden of disability and limits independence, ability to work and treatment adherence. Cognitive impairment is present in 40-70\% of people with MS in cross-sectional studies. Its prevalence and severity increase over time and as the disease enters its progressive phase. Cognitive impairment is already present in about a quarter of patients when they present with a CIS and it is present in more than half of patients 10 years from disease onset. Cognitive impairment in patients with CIS predicts conversion to MS and accumulation of physical disability. The pattern and severity of cognitive impairment (Box 3) are similar in PPMS and SPMS.

\section{The pattern of cognitive impairment}

Frank dementia is rare, and most patients experience mild to moderate cognitive impairment. A decline in IQ from estimated premorbid levels has been documented and impairment can be detected across many cognitive domains. Information processing and working memory are impaired early and more severely than would be expected from general cognitive blunting. For these reasons, tests of processing speed (e.g. the Paced Auditory Serial Addition Test or PASAT) are often used to screen for cognitive impairment. The combined effects of deficits in processing speed and working memory contribute to the impaired learning that is characteristic of the longterm memory problems experienced by $40-60 \%$ of people with MS, although deficits in retrieval and recognition are also present. Impaired complex attention and executive function are also common. Simple attention and basic verbal skills (naming and comprehension) are better preserved.

Decision-making, a complex skill that requires preserved executive function and memory, is also impaired in MS and this has an impact on employment, adherence to treatment and many aspects of everyday life. Mulhert and colleagues, using a gambling task, reported abnormalities in twice as many people with MS than controls. Slow deliberation

BOX 3 Pattern of cognitive impairment in multiple sclerosis

\footnotetext{
- Decreased IQ (from premorbid estimates)

- Slow processing speed and working memory

- Impaired learning

- Impaired executive function

- Preserved simple attention and basic language skills

- Impaired social cognition
} 
(related to executive function) and failure to gauge risk and make appropriate adjustments (related to memory) were the main difficulties experienced by the MS group. Slow deliberation correlated with medial prefrontal grey cortical changes, while poor risk adjustment was linked to hippocampal abnormalities. Impaired decision-making was present in otherwise cognitively preserved patients, but was more severe in those with cognitive impairment (Muhlert 2015).

Social cognition, a cluster of mental operations that underlie social interactions and that includes theory of mind and emotion recognition, has only recently received attention in people with MS. A systematic review and meta-analysis of 25 studies (Cotter 2016) has reported significant abnormalities in theory of mind and emotion recognition, particularly of negative emotions, in patients with MS compared with controls. Correlation between impaired social cognition and other cognitive deficits (processing speed, working memory and executive deficits) was inconsistent in these patients, and there was no correlation with depression. Impaired social cognition is more prominent when the disease enters its progressive phase.

\section{Factors that may influence cognitive performance}

Cognitive performance may be influenced by fatigue and depression, commonly present in MS. Performance on tests of attention, sustained attention and information processing speed is impaired in patients reporting fatigue. Depression has been linked to poor performance on a variety of tasks, but those requiring working memory and large cognitive processing capacity are particularly vulnerable to the effects of depression.

Close to $20 \%$ of people with MS use cannabis regularly and many others do so sporadically to control spasticity, pain or as a lifestyle choice (Feinstein 2015). Their cognitive performance may be altered across most domains, although quantitative effects are difficult to measure as the dose, potency and route of cannabis administration vary.

Cognitive reserve, as measured by pre-morbid IQ and years of education, is perhaps the most relevant factor in modulating the severity of cognitive impairment and may account, at least in part, for the variable impairment in MS patients with similar disease duration and disability.

\section{Brain pathology and cognitive impairment}

White and grey matter lesions and abnormalities in NABT cause disconnection in widespread networks relevant to cognition. Damage to cortico-subcortical connections results in slow processing speed, whereas damage to frontotemporal and frontoparietal networks results in impaired attention, memory and executive function. Cognitive impairment correlates with the volume of white and grey matter lesions, which can now be visualised using advanced MRI sequences (e.g. double inversion recovery or DIR). Cognitive impairment is also correlated with abnormalities in NABT. Detectable T1 and T2 white matter lesions when patients present with a CIS also predict future cognitive impairment.

It has proved more difficult to correlate specific cognitive deficits with regional brain pathology. A modest correlation has been reported between frontal white matter lesion load (Foong 1997) or frontal cortical complexity, a DTI-related measure (Muhlert 2013), and impaired executive function, suggesting that cognitive deficits result from damage to extensive networks, rather than localised pathology. Damage to white matter tracts that include the fornix, superior longitudinal fasciculus and forceps major appears to be particularly relevant in causing cognitive impairment, and damage to the posterior cingulate may result in disconnection in the default mode network, disturbing compensatory cognitive mechanisms. Cortical thinning, age, white matter lesion load and abnormalities in NABT (e.g. as detected with diffusion imaging) are the best predictors of cognitive impairment.

Changes in brain plasticity to maintain cognitive performance, so-called adaptive plasticity, have been studied in MS patients using fMRI in conjunction with attention and memory activation paradigms. Increased activation, often bilateral, in areas normally activated by a cognitive task, together with activation in commonly silent areas, have been documented early in the disease in cognitively intact patients. Similarly, increased functional connectivity in the default mode network and sensorimotor networks has been reported in cognitively preserved patients (Sumowski 2010), but as the disease progresses the increased activation/connectivity is no longer an effective compensatory mechanism and cognitive impairment becomes apparent. At this stage, the degree of increased activation/connectivity is correlated with lesion burden and with abnormalities in NABT.

\section{Treatment of cognitive impairment}

Disease-modifying therapies and cognition

Randomised controlled trials of DMTs tend to be underpowered or have too short follow-ups to provide strong evidence of cognitive improvement. However, available data suggest a beneficial effect of DMTs on cognition. Thus, interferon-beta-1a was found to improve processing speed, learning, visuospatial and executive function in a 2-year 
trial compared with placebo and the improvement persisted several years later (Patti 2009). Similar results have been reported for interferon-beta- $1 \mathrm{~b}$ (Lacy 2013). Benefits were greater when DMTs were administered early and in high doses. Greater benefits on cognition have been reported in small samples of patients treated with natalizumab (Weinstock-Guttman 2012). The reduction of inflammation with subsequent improvement in the efficiency of distributed networks is likely to account for the effect of DMTs on cognition.

\section{Other pharmacological treatments}

Symptomatic treatment with acetylcholinesterase inhibitors has produced variable results. The reported beneficial effects of donepezil have not been replicated and there is no evidence that memantine or rivastigmine have enhancing cognitive properties in people with MS.

Single-dose trials of methylphenidate (Harel 2009) and levoamphetamine (Benedict 2008) compared with placebo have reported improvements in attention and processing speed, suggesting that noradrenaline deficiency may contribute to cognitive impairment in MS. Regular administration of the stimulant lisdexamfetamine dimesylate (LDX), a medication used to treat attention-deficit hyperactivity disorder, has been reported to improve processing speed and memory (Morrow 2013).

\section{Cognitive rehabilitation}

A systematic review of 16 trials meeting required standards (O'Brian 2008) found some evidence of memory improvement using techniques based on visual imagery and context. Memory improvement has been associated with increased fMRI activation and improved connectivity in task-specific and contralateral areas, and recruitment of brain regions not normally activated by the memory task (Chiaravalloti 2015). This activation pattern is difficult to interpret, because of its similarity to the aberrant changes in brain plasticity present in cognitively impaired patients. More work is needed to replicate the reported improvement after memory rehabilitation to ascertain whether rehabilitation is possible for other cognitive functions and whether any gains can be maintained. Improvement in working memory after single transcranial magnetic stimulation (TMS) applied to the right dorsolateral prefrontal cortex has recently been reported, with simultaneous fMRI normalisation of the pattern of frontal hyperactivity (Hulst 2017).

\section{Conclusions}

Multiple sclerosis usually runs a relapsing-remitting course before entering a progressive phase, and new DMTs have reduced the frequency of relapses and the burden of disability, although it is uncertain whether they also limit the neurodegeneration process that is present from the early stages. MS has a high psychiatric morbidity and half of patients will experience depression and anxiety at some point in the illness. Brain pathology, together with psychosocial factors, determine the presence of depression. Psychosis and abnormalities of emotional expression are also increased, as is the prevalence of alcohol dependency.

Cognitive impairment is present in about half of patients in cross-sectional studies and it may occur early in the disease. Its early presence signals a poor prognosis. Cognitive impairment is mild to moderate in most cases. Processing speed, working memory, learning and executive functions are most severely impaired. Brain plasticity initially maintains cognition, but as the burden of pathology increases, cognitive impairment manifests. Cognitive reserve modulates deficits in cognition.

Disease-modifying therapies have a beneficial effect on depression and protect against cognitive impairment. Longer follow-up studies are needed to determine whether these protective effects are maintained. Cognitive neurorehabilitation and TMS hold promise, particularly if the progression of cognitive impairment can be halted by DMTs.

\section{References}

Andreassen OA, Harbo HF, Wang Y, et al (2015) Genetic pleiotropy between multiple sclerosis and schizophrenia but not bipolar disorder: differential involvement of immune-related gene loci. Molecular Psychiatry, 20: 207-14.

Bamer AM, Cetin K, Johnson KL, et al (2008) Validation study of prevalence and correlates of depressive symptomatology in multiple sclerosis. General Hospital Psychiatry, 30: 311-7.

Benedict RH, Munschauer F, Zarevics P, et al (2008) Effects of I-amphetamine sulfate on cognitive function in multiple sclerosis patients. Journal of Neurology, 255: 848-52.

Benros ME, Eaton WW, Mortensen PB (2014) The epidemiologic evidence linking autoimmune diseases and psychosis. Biological Psychiatry, 75: $300-6$.

Chiaravalloti ND, Genova HM, DeLuca J (2015) Cognitive rehabilitation in multiple sclerosis: the role of plasticity. Frontiers in Neurology, 6: 67

Cotter J, Firth J, Enzinger C (2016) Social cognition in multiple sclerosis: a systematic review and meta-analysis. Neurology, 87: 1727-36.

Cottrell S, Wilson S (1926) The affective symptomatology of disseminated sclerosis: a study of 100 cases. Journal of Neurological Psychopathology, 7: $1-30$

Ensari I, Motl RW, McAuley E, et al (2014) Patterns and predictors of naturally occurring change in depressive symptoms over a 30-month period in multiple sclerosis. Multiple Sclerosis, 20: 602-9.

Feinstein A (2002) An examination of suicidal intent in patients with multiple sclerosis. Neurology, 59: 674-8.

Feinstein A, Banwell E, Pavisian B (2015) What to make of cannabis and cognition in MS: in search of clarity amidst the haze. Multiple Sclerosis Journal, 21: 1755-60.

Feinstein A, du Boulay G, Ron MA (1992) Psychotic illness in multiple sclerosis: a clinical and magnetic resonance imaging study. British Journal of Psychiatry, 161: 680-5.
MCO answers

$1 \mathrm{c} \quad 2 \mathrm{~d} \quad 3 \mathrm{c} \quad 4 \mathrm{~b} \quad 5 \mathrm{e}$ 
Feinstein A, Magalhaes S, Richard JF, et al (2014) The link between multiple sclerosis and depression. Nature Reviews, Neurology, 10: 507-17.

Feinstein A, O'Connor P, Akbar N, et al (2010) Diffusion tensor imaging abnormalities in depressed multiple sclerosis patients. Multiple Sclerosis, 16: 189-96.

Fernie BA, Kollmann J, Brown RG (2015) Cognitive behavioural interventions for depression in chronic neurological conditions: a systematic review. Journal of Psychosomatic Research, 78: 411-9.

Fiest KM, Walker JR, Bernstein CN, et al (2016) Systematic review and meta-analysis of interventions for depression and anxiety in persons with multiple sclerosis. Multiple Sclerosis and Related Disorders, 5: 12-26.

Foong J, Rozewicz L, Quaghebeur G, et al (1997) Executive function in multiple sclerosis. The role of frontal lobe pathology. Brain, 120: 15-26.

Gobbi C, Rocca MA, Riccitelli G, et al (2014) Influence of the topography of brain damage on depression and fatigue in patients with multiple sclerosis. Multiple Sclerosis, 20: 192-201.

Gold S, Kern K, O'Connor M (2010) Smaller cornu ammonis, 2-3/dentate gyrus volumes and elevated cortisol in multiple sclerosis patients with depressive symptoms. Biological Psychiatry, 68: 553-9.

Harel Y, Appleboim N, Lavie M, et al (2009) Single dose of methylphenidate improves cognitive performance in multiple sclerosis patients with impaired attention process. Journal of Neurological Sciences, 276: 38-40.

Hulst HE, Goldschmidt T, Nitsche MA, et al (2017) rTMS affects working memory performance, brain activation and functional connectivity in patients with multiple sclerosis. Journal of Neurology, Neurosurgery and Psychiatry, 88: 386-94

Lacy M, Hauser M, Pliskin N, et al (2013) The effects of long-term interferon-beta- $1 \mathrm{~b}$ treatment on cognitive functioning in multiple sclerosis: a 16-year longitudinal study. Multiple Sclerosis, 19: 1765-72.

Marrie RA, Cohen J, Stuve 0, et al (2015) A systematic review of the incidence and prevalence of comorbidity in multiple sclerosis: overview. Multiple Sclerosis, 21: 263-81.

Minden SL, Feinstein A, Kalb RC, et al (2014) Evidence-based guideline: assessment and management of psychiatric disorders in individuals with MS: report of the Guideline Development Subcommittee of the American Academy of Neurology. Neurology, 82: 174-81.

Mohr DC, Hart SL, Fonareva I, et al (2006) Treatment of depression in patients with multiple sclerosis in neurological clinics. Multiple Sclerosis, 12: 204-8.

Morrow SA, Smerbeck A, Patrick K, et al (2013) Lisdexamfetamine dimesylate improves processing speed and memory in cognitively impaired MS patients: a phase II study. Journal of Neurology, 260: 489-97.

Muhlert N, Sethi V, Schneider T, et al (2013) Diffusion MRI-based cortical complexity alterations associated with executive function in multiple sclerosis. Journal of Magnetic Resonance Imaging, 38: 54-63.

Muhlert N, Sethi V, Cipolotti L, et al (2015) The grey matter correlates of impaired decision making in multiple sclerosis. Journal of Neurology Neurosurgery and Psychiatry, 86: 530-6.
Nigro S, Passamonti L, Riccelli R, et al (2015) Structural 'connectomic' alterations in the limbic system of multiple sclerosis patients with major depression. Multiple Sclerosis, 21: 1003-12.

Nygaard G. Walhovd K. Sowa P (2015) Cortical thickness and surface area relate to specific symptoms in early relapsing-remitting multiple sclerosis. Multiple Sclerosis, 21: 402-14.

O’Brien A, Chiaravalloti N, Goverover Y, et al (2008) Evidence-based cognitive rehabilitation for persons with multiple sclerosis: a review of the literature. Archives of Physical Medicine and Rehabilitation, 89: 761-9.

Passamonti L, Cerasa A, Liguori M, et al (2009) Neurobiological mechanisms underlying emotional processing in relapsing-remitting multiple sclerosis. Brain, 132: 3380-91.

Patten S, Svenson L, Metz L (2005) Psychotic disorders in MS: populationbased evidence of an association. Neurology, 65: 1123-5.

Patti F, Amato M, Bastianello S, et al (2009) Subcutaneous interferon beta-1a has a positive effect on cognitive performance in mildly disabled patients with relapsing remitting multiple sclerosis: 2-years results from the COGIMUS study. Therapeutic Advances in Neurological Disorder, 2 : $67-77$.

Pioro EP (2014) Review of Dextromethorphan $20 \mathrm{mg} /$ Quinidine $10 \mathrm{mg}$ (NUEDEXTA ${ }^{\circledR}$ ) for pseudobulbar affect, Neurology and Therapy, 3: 15-28.

Price A, Rayner L, Okon-Rocha E, et al (2011) Antidepressants for the treatment of depression in neurological disorders: a systematic review and meta-analysis of randomized control trials. Journal of Neurology, Neurosurgery and Psychiatry, 82: 914-23.

Rocca MA, Pravatà E, Valsasina P, et al (2015) Hippocampal-DMN disconnectivity in MS is related to WM lesions and depression. Human Brain Mapping, 36: 5051-63.

Scalfari A, Knappertz V, Cutter G, et al (2013) Mortality in patients with multiple sclerosis. Neurology, 81: 184-92.

Solomon A, Weinshenker B (2013) Misdiagnosis of multiple sclerosis: frequency, causes, effects, and prevention. Current Neurology and Neuroscience Reports, 13: 403

Strober LB, Arnett PA (2010) Assessment of depression in multiple sclerosis: development of a "trunk and branch" model. Clinical Neuropsycholy, 24: $1146-66$.

Sumowski JF, Wylie GR, Deluca J, et al (2010) Intellectual enrichment is linked to cerebral efficiency in multiple sclerosis: functional magnetic resonance imaging evidence for cognitive reserve. Brain, 133: $362-74$

Titulaer MJ, Höftberger R, lizuka T, et al (2014) Overlapping demyelinating syndromes and anti-N-methyl-D-aspartate receptor encephalitis. Annals of Neurology, 75: 411-28.

Weinstock-Guttman B, Galetta SL, Giovannoni G, et al (2012) Additional efficacy endpoints from pivotal natalizumab trials in relapsing-remitting MS. Journal of Neurology, 259: 898-905. 
MCOs

Select the single best option for each question stem

1 Multiple sclerosis (MS):

a is equally common in men and women

b usually runs a progressive course from the beginning

c is an immunological disease

$\mathrm{d}$ is a disease of the white matter of the brain

e requires a lumbar puncture to make the diagnosis.

2 The following symptoms are of little value in diagnosing depression in MS:

a feelings of guilt and disappointment

b sadness

c sense of failure d fatigue and disturbed sleep

e changes in appetite and weight.

3 In MS, emotional lability:

a is present in nearly every patient

$\mathrm{b}$ is commonly present at the onset of the disease

c is associated with executive function deficits

d does not respond to selective serotonin reuptake inhibitors (SSRIs)

$\mathrm{e}$ is more common in men.

4 The following cognitive domain tends to be preserved in MS:

a information processing speed

b naming and language comprehension

c working memory d complex attention

e executive function.

5 In MS, cognitive performance is unlikely to be influenced by:

a cannabis use

b depression

c cognitive reserve

d disease-modifying treatments (DMTs)

e acetylcholinesterase inhibitors. 